\title{
3D Crystallographic Imaging Using Laboratory-Based Diffraction Contrast Tomography (DCT)
}

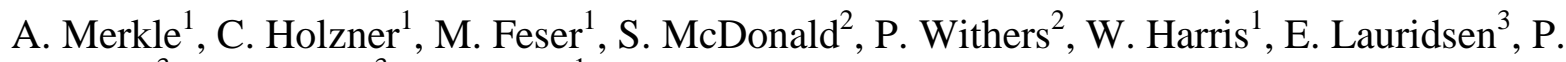
Reischig $^{3}$, H. Poulsen ${ }^{3}$, L. Lavery ${ }^{1}$

${ }^{1}$ Carl Zeiss X-ray Microscopy, Inc., Pleasanton, CA USA

${ }^{2}$ Manchester X-ray Imaging Facility, School of Materials, University of Manchester, Manchester M13 9PL, UK

${ }^{3}$ Xnovo Technology ApS, 4600, Køge, Denmark

Traditional X-ray tomography has, for some time, operated under a single absorption-based contrast mechanism. However, in recent years X-ray imaging has experienced a dramatic increase in the range of accessible imaging modalities - extending the classical absorption contrast with e.g. phase contrast, dark-field contrast, fluorescence, diffraction contrast, etc. Common for almost all such new imaging modalities are that they were developed at synchrotron facilities, and then - for some - have since been implemented on laboratory X-ray systems. [1,2]

Crystallographic imaging is primarily known from electron microscopy, and particularly the introduction of the electron back-scattering diffraction (EBSD) technique in the early 1990's, has made it a routine tool for research and/or development related to metallurgy, functional ceramics, semi-conductors, geology etc. The ability to image the grain structure in such materials is instrumental for understanding and optimization of material properties and processing. [3] However, the destructive nature of 3D EBSD prevents the technique from directly evaluating the microstructure (and grain-orientation) evolution when subject to either mechanical, thermal or other environmental conditions. Conversely, nondestructive X-ray imaging methods allow for such '4D' time dependent studies, but to date have been primarily the domain of a limited number of synchrotron facilities. [4]

Here we present the development and application of a new method, termed diffraction contrast tomography (DCT), to extend the capabilities of lab-based X-ray tomography systems to nondestructively analyze 3D crystallographic information of polycrystalline samples. The technique is implemented on a commercial laboratory X-ray microscope that utilizes a polychromatic divergent beam and a synchrotron-style detection system. During data collection, rotation and imaging of the sample yields a series of diffraction patterns generated by the sample crystallites when the Bragg condition is satisfied. The patterns are then reconstructed to yield grain orientation, center of mass, and size for a large number of grains.

This work will present a selection of results of laboratory DCT, including a beta titanium alloy sample and a microstructure of sintered copper spheres. Discussion of the implementation will include the boundary conditions and capabilities of the method, including coupling to in situ environments within the microscope or subjecting samples to extended time evolution experiments (across days, weeks, months). In addition, ways in which the DCT method can be correlatively coupled to related characterization techniques will also be examined, such as by employing focused ion beam serial sectioning for destructive, but complementary, characterization of the same volume following a DCT evolution experiment. 
[1] A. P. Merkle et al., Ascent of 3D X-ray Microscopy in the Laboratory, Microscopy Today, 21 (2013), p. 10

[2] E. Maire and P. Withers, Quantitative X-ray tomography, International Materials Review, 59 (2014) p. 1

[3] "Electron Backscatter Diffraction in Materials Science," ed. A. Schwartz et al., Springer US (2009)

[4] W. Ludwig et al., X-ray diffraction contrast tomography: a novel technique for threedimensional grain mapping of polycrystals. 1. Direct beam case, J. Appl. Crystallogr., 41 (2008), p. 302-309

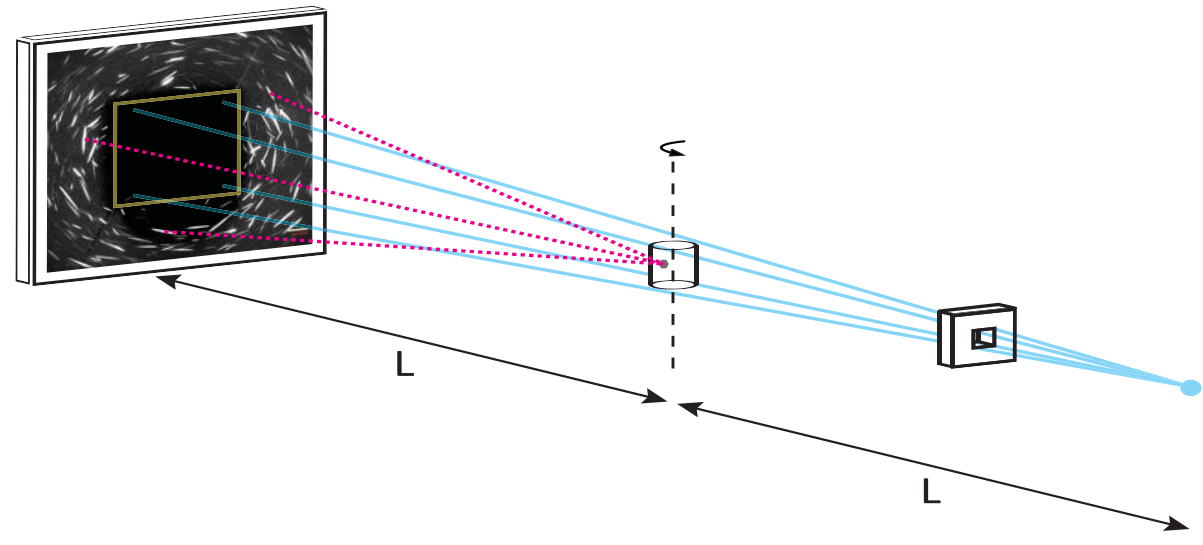

Figure 1. Experimental arrangement for lab DCT. A polychromatic source is located on the right, with X-rays proceeding through an aperture to illuminate the sample located in the center. A series of exposures are collected while the sample is rotated, creating diffraction patterns on the detector on the left, where the primary beam is blocked with a beam stop.

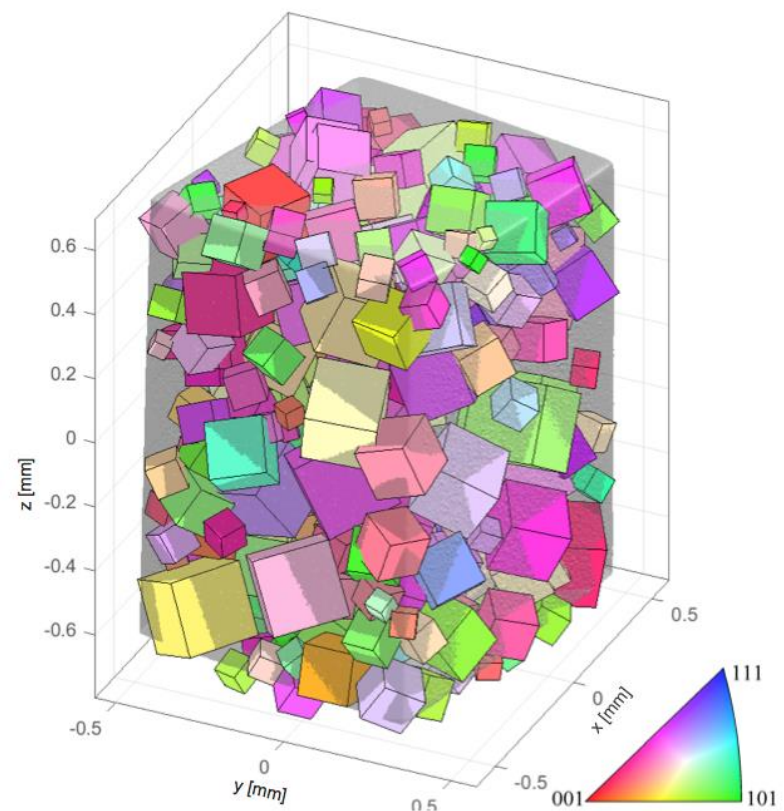

Figure 2. A cube plot representation of results from applying the DCT method to a beta titanium alloy sample. Each cube represents the location and relative size of an individual crystallite, with orientation denoted by color as shown in the inverse pole figure. 